\title{
Patient characteristics are important determinants of neurodevelopmental outcome at one year of age after neonatal and infant cardiac surgery
}

J. William Gaynor, MD, ${ }^{a}$ Gil Wernovsky, MD, ${ }^{b}$ Gail P. Jarvik, MD, PhD, ${ }^{c}$ Judy Bernbaum, MD, ${ }^{d}$ Marsha Gerdes, PhD, ${ }^{e}$ Elaine Zackai, MD, ${ }^{f}$ Alex S. Nord, BA, ${ }^{c}$ Robert R. Clancy, MD, ${ }^{g}$ Susan C. Nicolson, MD, ${ }^{h}$ and Thomas L. Spray, MD ${ }^{a}$

Supplemental material is available online.
From the Divisions of Cardiothoracic Surgery, ${ }^{\mathrm{a}}$ Pediatric Cardiology, ${ }^{\mathrm{b}}$ General Pediatrics, ${ }^{\mathrm{d}}$ Psychology, ${ }^{\mathrm{e}}$ Genetics, ${ }^{\mathrm{f}}$ Neuro$\log ,{ }^{,}$and Cardiothoracic Anesthesiology, ${ }^{\mathrm{h}}$ The Cardiac Center at The Children's Hospital of Philadelphia, Philadelphia, Pa; and the Department of Medicine (Medical Genetics), ${ }^{\mathrm{C}}$ University of Washington, Seattle, Wash.

Supported by a grant from the Fannie E. Rippel Foundation, an American Heart Association National Grant-in-Aid (9950480N), and grant HL071834 from the National Institutes of Health.

Read at the Eighty-sixth Annual Meeting of The American Association for Thoracic Surgery, Philadelphia, Pa, April 29-May 3, 2006.

Received for publication May 15, 2006; revisions received Oct 12, 2006; accepted for publication Oct 25, 2006.

Address for reprints: J. William Gaynor, MD, Division of Cardiothoracic Surgery, The Children's Hospital of Philadelphia, 34th and Civic Center Blvd, Suite 8527, Philadelphia, PA 19104 (E-mail: gaynor@email.chop. edu).

J Thorac Cardiovasc Surg 2007;133:1344-53 $0022-5223 / \$ 32.00$

Copyright () 2007 by The American Association for Thoracic Surgery

doi:10.1016/j.jtcvs.2006.10.087
Objective: Many studies of neurodevelopmental outcomes after neonatal and infant cardiac surgery have focused on potentially modifiable risk factors for adverse outcomes, primarily intraoperative management strategies and the use of deep hypothermic circulatory arrest. There is increasing evidence that patient-specific factors are more important determinants of outcome.

Methods: We investigated predictors of neurodevelopmental outcomes at 1 year of age after neonatal and infant cardiac surgery in a subgroup of infants enrolled in a prospective study of apolipoprotein E (APOE) genotype and neurodevelopmental outcome. Children with a variety of 2-ventricle cardiac defects repaired with only 1 operation with cardiopulmonary bypass and no more than 1 episode of deep hypothermic circulatory arrest were included. Neurodevelopmental outcomes at 1 year of age included the Bayley Scales of Infant Development-II, which yield 2 indices, the Mental Developmental Index and the Psychomotor Developmental Index.

Results: Two hundred forty-seven infants underwent surgical repair between October 1998 and April 2003 with 1 hospital death and 3 deaths before 1 year of age. Neurodevelopmental evaluation was performed in 188 (77\%) of 243 survivors, including 56 patients with tetralogy of Fallot, 39 with transposition of the great arteries with intact ventricular septum, 34 with ventricular septal defects, and 59 with other defects. The median age at operation was 56 days (1-186 days), including $72(38 \%)$ neonates. Confirmed or suspected genetic syndromes were present in 59 (31\%) of 188 infants. Deep hypothermic circulatory arrest was used in 67 (35\%) infants with a median duration of 34 minutes (1-80 minutes). For the entire cohort, the mean Mental Developmental Index was $90.6 \pm 14.9$ and the mean Psychomotor Developmental Index was $81.6 \pm 17.2$. For patients without genetic syndromes, the mean Mental Developmental Index was $93.7 \pm 13.6$ and the mean Psychomotor Developmental Index was $85.1 \pm 14.6$. For the entire cohort, predictors of lower scores for both the Mental Developmental Index and Psychomotor Developmental Index were presence of a confirmed or suspected genetic syndrome, lower birth weight, and presence of the APOE $\epsilon 2$ allele (all $P<.04$ ). Black race was associated with higher scores on the Psychomotor Developmental Index $(P=.018)$. Lower nasopharyngeal temperature during cardiopulmonary bypass was associated with a lower score on the Psychomotor Developmental Index $(P=.03)$ and was the only intraoperative factor that was a significant predictor of either the Mental or Psychomotor Developmental Index.

Conclusions: The strongest predictors of a worse neurodevelopmental outcome at 1 year of age were patient-specific factors including presence of a genetic syndrome, low birth weight, and presence of the APOE $\epsilon 2$ allele. Patient-specific factors eclipsed the use and duration of deep hypothermic circulatory arrest as predictors of worse neurodevelopmental outcomes. 
$\mathrm{T}$ here has been increasing recognition of adverse neurodevelopmental outcomes in some survivors of neonatal and infant cardiac surgery. Many previous observational studies and neuroprotective trials have focused on potentially modifiable risk factors, particularly intraoperative management strategies. The findings of these studies have led many institutions to alter their intraoperative management strategies, particularly avoidance of deep hypothermic circulatory arrest (DHCA). However, review of these studies suggests that factors other than intraoperative management strategies may be more important determinants of neurodevelopmental outcomes for many children. The Boston Circulatory Arrest Study (BCAS) provides the most comprehensive overview of neurodevelopmental outcomes after infant cardiac surgery. ${ }^{1-4}$ In this study, infants undergoing the arterial switch procedure (1988-1992) for transposition of the great arteries (TGA) with or without a ventricular septal defect (VSD) were prospectively randomized to an operative support strategy of either primarily DHCA or primarily continuous low-flow cardiopulmonary bypass (CPB). At 8 years of age, neurodevelopmental status for the cohort as a whole was below expectations for many domains, including academic achievement, fine motor function, and higher-order language skills, regardless of use of DHCA or low-flow CPB. ${ }^{3}$ Longer postoperative length of stay in the cardiac intensive care unit at the time of the initial operation was an independent risk factor for lower IQ scores at 8 years. ${ }^{5}$ The lack of a treatment group effect for many outcomes and the finding that prolonged length of stay was associated with a worse outcome at 8 years suggest that factors other than intraoperative management strategies may be important determinants of neurodevelopment outcome. ${ }^{3}$

The current study was undertaken to evaluate the relative contributions of a variety of patient-specific factors (eg, gestational age, ethnicity, presence of a genetic syndrome), as well as intraoperative management strategies, as predictors of neurodevelopmental outcomes at 1 year of age after neonatal and infant cardiac surgery. The study uses a subgroup of infants enrolled in a prospective study evaluating apolipoprotein $\mathrm{E}(A P O E)$ genotype as a risk factor for adverse neurodevelopmental outcomes after neonatal and infant cardiac surgery. ${ }^{6}$ To minimize the potential confounding effects of chronic cyanosis and multiple operations, the current study evaluated children with a variety of 2-ventricle congenital heart defects (CHD) undergoing complete repair with only one operation with $\mathrm{CPB}$ with or without DHCA.

\section{Materials and Methods Sample}

This study constitutes a secondary analysis of a subgroup of patients enrolled in a prospective trial assessing the effects of polymorphisms of the APOE gene on neurodevelopmental outcomes in patients 6 months of age or less undergoing surgical

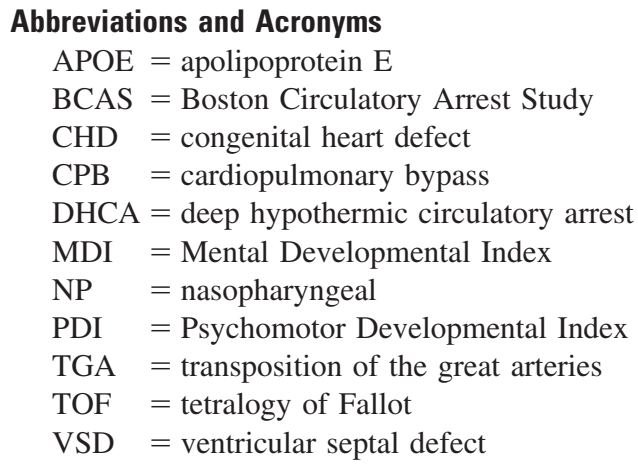

repair for CHD. ${ }^{6}$ Patients 6 months of age or less undergoing CPB with or without DHCA for repair of CHD were eligible. Exclusion criteria included (1) multiple congenital anomalies, (2) recognizable genetic or phenotypic syndrome other than chromosome 22q11 microdeletions, and (3) language other than English spoken in the home. The current study evaluated infants who underwent repair of a variety of 2-ventricle cardiac defects with CPB with or without DHCA. Patients undergoing more than one operation with CPB or more than one episode of DHCA were excluded. The study was approved by the Institutional Review Board at The Children's Hospital of Philadelphia. Informed consent was obtained from the parent or guardian.

\section{Operative Management}

Surgery was performed by 5 cardiac surgeons with a dedicated team of cardiac anesthesiologists. Alpha-stat blood gas management was used. Pump flow rates were not standardized for this study. In general, during normothermic or mild hypothermic CPB (nasopharyngeal $[\mathrm{NP}]$ temperature $>28^{\circ} \mathrm{C}$ ), the pump flow rate was maintained at 100 to $150 \mathrm{~mL} \cdot \mathrm{kg}^{-1} \cdot \mathrm{min}^{-1}$ to achieve a mean arterial pressure of 30 to $55 \mathrm{~mm} \mathrm{Hg}$. When moderate hypothermia (NP temperature $22^{\circ} \mathrm{C}-28^{\circ} \mathrm{C}$ ) was used, the pump flow rate was maintained at $100 \mathrm{~mL} \cdot \mathrm{kg}^{-1} \cdot \mathrm{min}^{-1}$ with a target mean arterial pressure greater than $30 \mathrm{~mm} \mathrm{Hg}$. For deep hypothermic continuous $\mathrm{CPB}$ (NP temperature $<22^{\circ} \mathrm{C}$ ), a pump flow rate of 25 to $50 \mathrm{~mL}$. $\mathrm{kg}^{-1} \cdot \min ^{-1}$ was used. These were general guidelines that were modified according to the clinical situation. DHCA was used at the surgeon's discretion. Before DHCA, patients underwent core cooling, with topical hypothermia of the head, to an NP temperature of $18^{\circ} \mathrm{C}$. Modified ultrafiltration was performed in all patients. Postoperatively, patients were managed in the cardiac intensive care unit by a dedicated team of cardiologists and intensivists.

\section{Data Collection}

Preoperative factors including gestational age, birth head circumference, birth weight, Apgar scores, and preoperative intubation were obtained from birth and hospital records. Weight, age at operation, and type of operation were recorded along with perfusion data, including CPB time, aortic crossclamp time, and duration of DHCA. Total support time was calculated as CPB time plus DCHA time. 


\section{APOE Genotype Determination}

Whole blood or a buccal swab was obtained before the operation and stored at $4^{\circ} \mathrm{C}$. Genomic DNA was prepared and used to determine $A P O E$ genotypes using a previously published method. ${ }^{7}$

\section{One-year Neurodevelopmental Examination}

Children were scheduled for their 1-year follow-up visit as they approached 1 year of age. A 2-week window was established so that children were seen at 12 months \pm 2 weeks. Children born before 37 weeks of gestation were seen at their corrected age. The developmental assessment was performed first, followed by medical history, physical, and neurologic examinations. The developmental assessment included the Bayley Scales of Infant Development-II, which yields scores on two indices: the Psychomotor Development Index (PDI) and the Mental Developmental Index (MDI). The medical evaluation included interim health history and physical examination. The child's ethnicity and the familial socioeconomic status were assessed by parental report according to the Hollingshead scale. ${ }^{8}$ Ethnicity was classified as Asian/Pacific Islander, black, Hispanic, American Indian, other, or white. Neuromuscular examination included neuromuscular tone, symmetry, strength and skills, reflexes, and bulbar function. Children were classified as "normal" or "abnormal/suspect." Growth measurements included weight, length, and head circumference. Patients were also evaluated by a genetic dysmorphologist. Chromosomal analysis and testing for microdeletions of chromosome 22q11 were performed as indicated. Neonatal recognition of dysmorphic features may be difficult; therefore, some patients were enrolled in whom the diagnosis of a genetic syndrome was not made until the 1 -year evaluation. Patients were classified as having no definite genetic syndrome or chromosomal abnormality ("normal") or as having a definite or suspected genetic syndrome or chromosomal abnormality ("abnormal/suspect").

\section{Data Analysis and Statistical Methods}

Data are presented as either median (range) or mean \pm standard deviation, as appropriate. All predictors listed in Table 1 were tested by linear regression for univariate prediction of MDI, PDI, and head circumference, and logistic regression for prediction of neuromuscular outcome ( 0 = "normal," 1 = "abnormal/suspect"). Estimates for logistic regression models are given as log odds. Patients were classified according to their primary cardiac diagnosis. The most common diagnoses were tetralogy of Fallot (TOF), TGA, and VSD. Patients with other defects were coded as "other." Subjects were grouped by APOE genotype into the $\epsilon 2$ group $(\epsilon 2 \epsilon 2$ and $\epsilon 3 \epsilon 2$ ), the $\epsilon 3$ group $(\epsilon 3 \epsilon 3)$, and the $\epsilon 4$ group $(\epsilon 3 \epsilon 4$ and $\epsilon 4 \epsilon 4)$. The $\epsilon 2 \epsilon 4$ subjects were excluded from the analyses because the $\epsilon 2$ and $\epsilon 4$ alleles are opposing in their effects in some conditions, such as Alzheimer disease. Categorical variables were coded as dummy variables, with the most common category as the reference group and the overall model $P$ value used. For each outcome, all predictors with a marginal $P<.1$ were considered in a separate forward stepwise logistic regression to determine a model for prediction of that outcome. Stepwise model results are given for predictors with $P$ values $<.05$. Explained variance for all models was calculated by adjusted $r^{2}$ values. Use of DHCA was also considered separately from the quantitative variable duration of DHCA. All anal- yses used SPSS 10.0 for Windows (SPSS, Inc, Chicago, Ill) and the R statistical software environment.

\section{Results \\ Study Population}

Between October 1998 and April 2003, 675 eligible neonates and infants underwent cardiac surgery and $550(81 \%)$ enrolled in the APOE study. Of these 550 patients, 247 met the criteria of 2-ventricle CHD repaired with only one operation with $\mathrm{CPB}$ and no more then one episode of DHCA. There was 1 hospital death ( $0.4 \%$ mortality) with 3 additional deaths before 1 year of age. One-hundred eightyeight $(77 \%)$ of the 243 eligible patients returned and completed the 1-year evaluation. Fifty-five patients were alive at 1 year but did not return for evaluation.

Preoperative and intraoperative characteristics were compared for the 188 patients who returned and the 55 patients who did not return (Table E1). There were no differences in gender, ethnicity, $A P O E$ genotype, or cardiac diagnosis between the groups. All patients who were the product of a multiple gestation returned for 1-year evaluation. There were differences in some intraoperative variables between the two groups (Table E1). Patients who did not return were older at the time of operation and had shorter $\mathrm{CPB}$ and total support times. Among the patients undergoing continuous $\mathrm{CPB}$, the lowest NP temperature was less than $25^{\circ} \mathrm{C}$ in $37 \%$ of the patients and less then $20^{\circ} \mathrm{C}$ in $19 \%$ of the patients. Use and duration of DHCA was not significantly different between the two groups.

In the cohort of 188 patients who returned for 1-year evaluation, there were 85 female and 103 male patients. The median gestational age was 39 weeks (range, 28-42 weeks) and the median birth weight was $3175 \mathrm{~g}$ (range, 739-5140 g). Thirty-one (16\%) patients were less than 37 weeks' gestational age. Birth weight was less than $2250 \mathrm{~g}$ in $18(10 \%)$ patients. There were 4 patients of Asian/Pacific Island ethnicity, 23 black patients, 6 Hispanic patients, 136 white patients, and 19 of other ethnic origin. TOF was present in 56 patients, TGA with intact ventricular septum in 39 patients, VSD in 34 , and a variety of other 2 -ventricle cardiac defects in the remaining 59 patients.

The median age at operation was 56 days (range, 1-186 days). Surgical intervention was performed on $72(38 \%)$ patients as neonates (age $\leq 30$ days). The median weight at the time of operation was $3.9 \mathrm{~kg}$ (range, $1.7-7.7 \mathrm{~kg}$ ), and 8 (4\%) patients were less than $2.25 \mathrm{~kg}$ at the time of the operation. The median hematocrit after hemodilution on bypass was $27 \%$ (range, $17 \%-39 \%$ ) and was less than $25 \%$ in 46 patients. DHCA was used in $67(36 \%)$ patients (Figure 1). When DHCA was used, the median lowest NP temperature was $18^{\circ} \mathrm{C}$ (range, $15^{\circ} \mathrm{C}-28^{\circ} \mathrm{C}$ ). The median duration of cooling for patients undergoing DHCA was 15 minutes (range, 5-22 minutes). The median duration of DHCA was 34 minutes (range, 1-80 minutes). When DHCA was not used, 
TABLE 1. Single covariate analysis

\begin{tabular}{|c|c|c|c|c|c|c|c|c|c|c|c|c|c|}
\hline & \multirow[b]{2}{*}{$\mathbf{n}$} & \multicolumn{3}{|c|}{ MDI } & \multicolumn{3}{|c|}{ PDI } & \multicolumn{3}{|c|}{$\begin{array}{c}\text { Neuromuscular } \\
\text { examination }\end{array}$} & \multicolumn{3}{|c|}{ Head circumference } \\
\hline & & $\boldsymbol{\beta}$ & SE & $P$ & $\boldsymbol{\beta}$ & SE & $\boldsymbol{P}$ & $\boldsymbol{\beta}$ & SE & $\boldsymbol{P}$ & $\boldsymbol{\beta}$ & SE & $P$ \\
\hline \multicolumn{14}{|l|}{ Preoperative covariates } \\
\hline Gender & 188 & -1.936 & 2.184 & .376 & 0.348 & 2.533 & .891 & -0.034 & 0.336 & .920 & 1.281 & 0.236 & .000 \\
\hline Ethnicity (ref: white) & 188 & & & .964 & & & .056 & & & .092 & & & .145 \\
\hline Socioeconomic status & 188 & 1.591 & 0.991 & .110 & -0.155 & 1.155 & .894 & -0.136 & 0.149 & .361 & 0.226 & 0.115 & .050 \\
\hline Gestational age (wk) & 188 & 1.472 & 0.434 & .001 & 1.455 & 0.507 & .005 & -0.177 & 0.066 & .007 & 0.095 & 0.051 & .068 \\
\hline Multiple gestation $(\mathrm{Y} / \mathrm{N})$ & 187 & -13.362 & 4.757 & .006 & -9.124 & 5.590 & .104 & 0.701 & 0.669 & .295 & 0.099 & 0.562 & .860 \\
\hline Delivery (cesarean section vs vaginal) & 186 & 3.450 & 2.366 & .146 & 4.628 & 2.716 & .090 & -0.465 & 0.355 & .190 & -0.492 & 0.273 & .073 \\
\hline Apgar at $1 \mathrm{~min}$ & 184 & 0.968 & 0.659 & .144 & 2.031 & 0.754 & .008 & -0.334 & 0.102 & .001 & 0.111 & 0.077 & .152 \\
\hline Apgar at $5 \mathrm{~min}$ & 184 & 1.560 & 1.152 & .177 & 3.238 & 1.321 & .015 & -0.488 & 0.195 & .012 & 0.240 & 0.134 & .075 \\
\hline $\begin{array}{l}\text { Confirmed or suspected genetic } \\
\text { syndrome }\end{array}$ & 188 & -9.681 & 2.226 & .000 & -11.099 & 2.579 & .000 & 1.553 & 0.355 & .000 & -0.437 & 0.269 & .106 \\
\hline$A P O E 2$ genotype (ref: $\epsilon 33$ ) & 186 & & & .016 & & & .014 & & & .092 & & & .278 \\
\hline Weight at birth (gm) & 187 & 0.007 & 0.002 & .000 & 0.006 & 0.002 & .001 & -0.001 & 0.000 & .001 & 0.001 & 0.000 & .000 \\
\hline Head circumference at birth $(\mathrm{cm})$ & 183 & 1.397 & 0.492 & .005 & 1.259 & 0.583 & .032 & -0.196 & 0.078 & .012 & 0.410 & 0.050 & .000 \\
\hline Prostaglandin inf & 186 & 3.384 & 2.450 & .169 & 2.009 & 2.851 & .482 & -0.474 & 0.401 & .238 & -0.036 & 0.285 & .899 \\
\hline Preoperative intu & 186 & -3.188 & 2.554 & .214 & -4.479 & 2.955 & .131 & 0.129 & 0.075 & .087 & -0.302 & 0.296 & .310 \\
\hline Cardiac diagnos & 188 & & & .397 & & & .371 & & & .054 & & & .632 \\
\hline Preoperative length of stay $(d)$ & 188 & -0.364 & 0.220 & .101 & -0.635 & 0.253 & .013 & 0.105 & 0.048 & .029 & -0.028 & 0.026 & .274 \\
\hline \multicolumn{14}{|l|}{ Operative covariates } \\
\hline Age at operation (d) & 188 & -0.002 & 0.020 & .914 & 0.004 & 0.023 & .852 & -0.004 & 0.003 & .198 & 0.000 & 0.002 & .928 \\
\hline Weight at operation $(\mathrm{kg})$ & 188 & 1.740 & 0.797 & .030 & 2.342 & 0.918 & .012 & -0.426 & 0.143 & .002 & 0.332 & 0.090 & .000 \\
\hline Surgeon (ref: 3) & 188 & & & .388 & & & .379 & & & .820 & & & .548 \\
\hline pport time $[\mathrm{CPB}+\mathrm{DHCA}](\mathrm{min})$ & 188 & -0.001 & 0.025 & .976 & -0.039 & 0.029 & .183 & 0.002 & 0.004 & .623 & -0.006 & 0.003 & .035 \\
\hline e $(\min )$ & 188 & 0.017 & 0.023 & 0.474 & -0.018 & 0.027 & .517 & 0.000 & 0.004 & .930 & -0.003 & 0.003 & .246 \\
\hline DHCA time (min) & 188 & -0.117 & 0.060 & .053 & -0.107 & 0.070 & .125 & 0.012 & 0.009 & .160 & -0.015 & 0.007 & .038 \\
\hline DHCA ( & 188 & -5.206 & 2.242 & .021 & -4.790 & 2.609 & .068 & 0.697 & 0.341 & .041 & -0.542 & 0.261 & .039 \\
\hline Hematocrit after hemodilution (\%) & 188 & 0.249 & 0.263 & .344 & 0.385 & 0.304 & .206 & -0.012 & 0.040 & .763 & -0.051 & 0.030 & .096 \\
\hline Cooling time (min) & 188 & -0.134 & 0.179 & .457 & -0.367 & 0.206 & .076 & 0.037 & 0.028 & .181 & -0.051 & 0.020 & .013 \\
\hline Lowest NP temperature $\left({ }^{\circ} \mathrm{C}\right)$ & 188 & 0.234 & 0.205 & .256 & 0.616 & 0.234 & .009 & -0.078 & 0.035 & .024 & 0.072 & 0.023 & .002 \\
\hline Delayed sternal closure (Y/N) & 188 & -6.650 & 4.266 & .121 & -6.171 & 4.948 & .214 & 0.652 & 0.597 & .275 & -0.866 & 0.494 & .081 \\
\hline ECMO (Y/N) & 188 & 7.000 & 10.605 & .510 & 1.425 & 12.288 & .908 & 16.679 & 1029.121 & .987 & -0.398 & 1.231 & .747 \\
\hline Postoperative length of stay (d) & 188 & -0.286 & 0.100 & .005 & -0.462 & 0.113 & .000 & 0.096 & 0.028 & .001 & -0.022 & 0.012 & .068 \\
\hline Total length of stay $(d)$ & 188 & -0.247 & 0.082 & .003 & -0.405 & 0.092 & .000 & 0.082 & 0.022 & .000 & -0.019 & 0.010 & .055 \\
\hline
\end{tabular}

For each outcome, single covariates with $P \leq 0.1$ are shown in boldface. $A P O E$, Apolipoprotein $\mathrm{E}$; $\beta$, regression coefficient; $C P B$, cardiopulmonary bypass; $D H C A$, deep hypothermic circulatory arrest; ECMO, extracorporeal membrane oxygenation; $M D I$, mental developmental index; NP, nasopharyngeal; $P D I$, psychomotor developmental index; $S E$, standard error; TGA/IVS, transposition of the great arteries with intact ventricular septum; TOF, tetralogy of Fallot; $V S D$, ventricular septal defect; $Y / N$, yes/no.

the median NP temperature was $26^{\circ} \mathrm{C}$ (range, $\left.17^{\circ} \mathrm{C}-37^{\circ} \mathrm{C}\right)$. The median duration of total support was 79 minutes (range, 24-242 minutes).

\section{One-year Neurodevelopmental Evaluation}

For the entire cohort $(\mathrm{n}=188)$, the mean MDI was $90.6 \pm$ 14.9 and the mean PDI was $81.6 \pm 17.2$. For patients without genetic syndromes $(n=129)$, the mean MDI was $93.7 \pm 13.6$ and the mean PDI was 85.1 \pm 14.6. In the general population, mean MDI and PDI scores are 100 with a normal distribution. In this cohort, the distributions of scores for both the MDI and PDI are shifted leftward (Figure 2). The PDI is more severely affected than the MDI.
The MDI was 70 or less ( 2 standard deviations below the mean) in 17 (9\%) patients and the PDI was 70 or less in 42 $(22 \%)$ patients. Potential predictors of the MDI and PDI are listed in Table 1, as well as their univariate statistical significance. Stepwise logistic regression for MDI identified a model containing lower birth weight, presence of a confirmed or suspected genetic syndrome, and presence of the $A P O E \epsilon 2$ allele as significant predictors a lower MDI score (Table 2). For the PDI, stepwise logistic regression identified a model containing lower birth weight, presence of a confirmed or suspected genetic syndrome, the $A P O E \in 2$ allele, lower NP temperature, and longer postoperative 


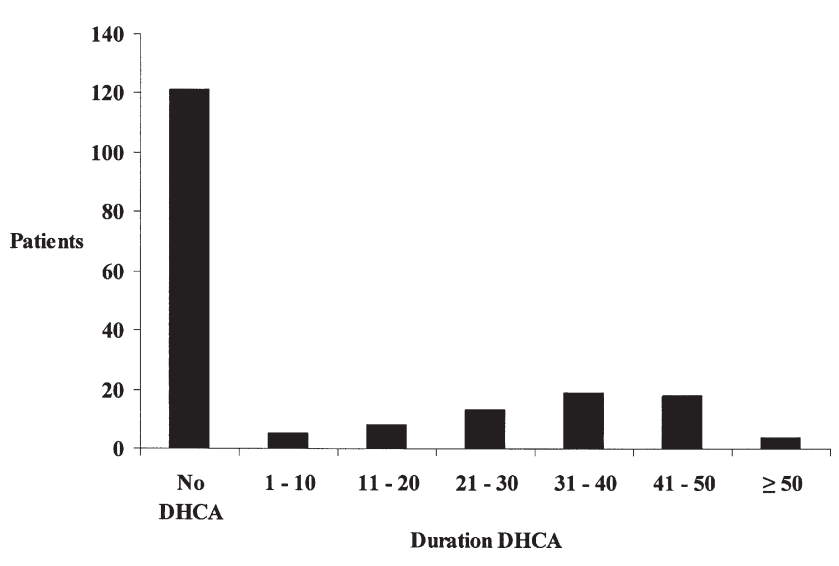

Figure 1. Bar graph showing use and duration of DHCA. The $x$-axis is DHCA duration in 10-minute increments. The $y$-axis is number of patients. DHCA, deep hypothermic circulatory arrest.

length of stay as significant predictors of a lower PDI score (Table 3). Black race was associated with higher scores on the PDI. Lower birth weight, presence of a genetic syndrome, and the APOE $\epsilon 2$ allele were predictors of lower scores for both the MDI and PDI. The distribution of MDI and PDI scores for patients with one or more of these risk factors compared with patients with no risk factor is shown in Figure E1. Patients with very low scores on both the MDI and PDI are more common in the group with at least one risk factor. Overall, patient-specific factors (gender, ethnicity, birth weight, birth head circumference, Apgar score at 1 minute, Apgar score at 5 minutes, genetic syndrome, $A P O E$ genotype) explained more of the variability in the MDI $(13.0 \%$ vs $5.3 \%)$ and the PDI $(20.7 \%$ vs $7.6 \%)$ than did intraoperative factors (weight at surgery, cooling time, DHCA time, CPB time, lowest NP temperature, hematocrit). Inclusion of other variables did not improve the pre-

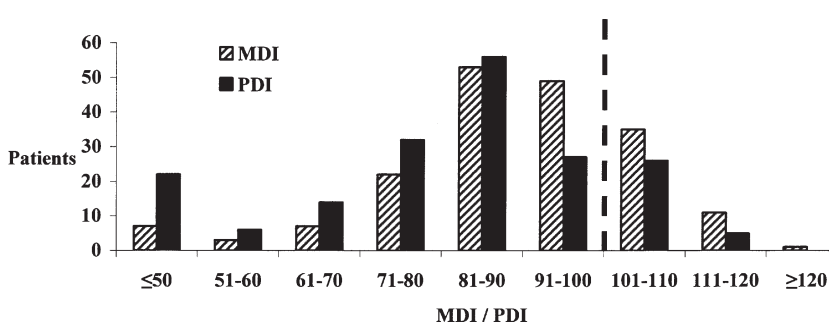

Figure 2. Bar graph showing distribution of MDI and PDI scores. The $x$-axis is MDI/PDI score in 10-point increments. The $y$-axis is number of patients. In the general population, MDI and PDI scores are normally distributed with a mean of 100 and a standard deviation of 15. In this cohort, the distribution is shifted to the left, indicating worse performance. The PDI is more severely affected than the MDI. MDI, Mental Developmental Index; PDI, Psychomotor Developmental Index.
TABLE 2. Predictors of MDI $(\mathbf{n}=\mathbf{1 8 8})$

\begin{tabular}{lrcrr}
\hline & Estimate & SE & \multicolumn{1}{c}{$\boldsymbol{t}$} & $\boldsymbol{P r}(>|t|)$ \\
\hline (Intercept) & 79.9084 & 5.4236 & 14.733 & .0000 \\
Birth weight & 0.0051 & 0.0016 & 3.212 & .0016 \\
Presence of confirmed or & -6.0820 & 2.2634 & -2.687 & .0079 \\
$\quad$ suspected genetic syndrome & & & & \\
APOE $\epsilon 2$ & -6.9136 & 3.1758 & -2.177 & .0309
\end{tabular}

$M D I$, Mental Developmental Index; $S E$, standard error; $A P O E$, apolipoprotein $\mathrm{E}$

dictive accuracy of the models for either MDI or PDI. The final, and best, models (Tables 2 and 3) for both MDI and PDI accounted for less than $30 \%$ of the variance in the scores, suggesting that unrecognized factors are important determinants of outcome.

Results of the neuromuscular examination were abnormal or suspect in $48(26 \%)$ patients. The most common abnormality on neuromuscular examination was hypotonia. Potential predictors of the neuromuscular examination are listed in Table 1, as well as their univariate statistical significance. Stepwise logistic regression identified a model containing lower birth weight, lower Apgar score at 1 minute, presence of a confirmed or suspected genetic syndrome, and longer postoperative length of stay as significant predictors of an abnormal or suspect neuromuscular examination (Table 4). Black race and other race were significantly associated with better performance on the neuromuscular examination.

Head circumference at 1 year was also assessed as a surrogate for brain growth. The median head circumference was $45.5 \mathrm{~cm}$ (range, 40.3-50.0 cm). Microcephaly (head circumference percentile $\leq 5 \%)$ was present in $44(23 \%)$ of 188 patients. Potential predictors of the head circumference at 1 year are listed in Table 1 , as well as their univariate statistical significance. Stepwise logistic regression identified a model containing male gender, head circumference at birth, higher Apgar scores at 5 minutes, and higher lowest NP temperature as significant predictors of larger head

TABLE 3. Predictors of PDI $(\mathbf{n}=188)$

\begin{tabular}{lrccc}
\hline & Estimate & SE & $\boldsymbol{t}$ & $\boldsymbol{P r}(>|\boldsymbol{t}|)$ \\
\hline (Intercept) & 51.1613 & 9.0248 & 5.6690 & .0000 \\
Birth weight & 0.0042 & 0.0018 & 2.3960 & .0177 \\
Black race & 11.6915 & 3.6481 & 3.2050 & .0016 \\
Presence of confirmed or & -8.0508 & 2.5063 & -3.2120 & .0016 \\
$\quad$ suspected genetic syndrome & & & & \\
APOE $\epsilon 2$ & -8.9199 & 3.5954 & -2.4810 & .0141 \\
Lowest NP temperature & 0.4802 & 0.2189 & 2.1930 & .0297 \\
Postoperative LOS & -0.2521 & 0.1062 & -2.3730 & .0188 \\
\hline
\end{tabular}

$P D I$, Psychomotor Developmental Index; $S E$, standard error; $A P O E$, apolipoprotein $\mathrm{E} ; N P$, nasopharyngeal; $L O S$, length of stay. 
TABLE 4. Predictors of abnormal or suspect neuromusculmination $(\mathbf{n}=\mathbf{1 8 8})$

\begin{tabular}{lrrrr}
\hline & Estimate & SE & \multicolumn{1}{c}{$\boldsymbol{t}$} & $\boldsymbol{P r}(>|\boldsymbol{t}|)$ \\
\hline (Intercept) & 5.5013 & 1.9466 & 2.8260 & .0047 \\
Birth weight & -0.0011 & 0.0004 & -3.0120 & .0026 \\
APGAR at 1 min & -0.3123 & 0.1177 & -2.6520 & .0080 \\
Black race & -3.3821 & 1.3561 & -2.4940 & .0126 \\
Other race & -1.8728 & 0.9440 & -1.9840 & .0473 \\
Presence of confirmed & 1.5055 & 0.4419 & 3.4070 & .007 \\
$\quad$ & & & & \\
$\quad$ or suspected & & & & \\
Ponetic syndrome & 0.0772 & 0.0353 & 2.1880 & .0287
\end{tabular}

$S E$, Standard error; LOS, length of stay.

circumference at 1 year of age (Table 5). Vaginal delivery was associated with a smaller head circumference at 1 year.

\section{Discussion}

The current study demonstrates that patient-specific factors such as birth weight, ethnicity, and the presence of a genetic syndrome are important determinants of neurodevelopmental outcome after neonatal and infant cardiac surgery. These patient-specific factors explain considerably more of the variability in outcomes at 1 year of age than do intraoperative management strategies, such as the use and duration of DHCA and hematocrit during CPB. In particular, neither the use nor the duration of DHCA was associated with a worse outcome at 1 year of age. However, the median duration of DHCA in the current study was 34 minutes (Figure 1).

Overall scores for both the MDI and the PDI are lower than population norms. Consistent with previous studies, motor skills as assessed by the PDI and the neuromuscular examination are more significantly impaired after infant cardiac surgery than is cognitive function assessed by the MDI. ${ }^{9-11}$ Interestingly, black infants performed better on both the PDI and neuromuscular examination than did infants of other ethnic groups. This finding is consistent with previous studies demonstrating that, among the general population, scores for black infants on the PDI at 1 year of age tend to be higher than those for other ethnic groups. ${ }^{12,13} \mathrm{It}$ is worrisome that microcephaly, suggesting impairment of brain growth, was identified in more than $20 \%$ of the cohort at 1 year of age.

Many studies of neurodevelopmental outcome after cardiac surgery have focused on intraoperative management strategies. ${ }^{4-9-11}$ This focus is understandable inasmuch as these strategies can be modified and thus there is an opportunity to potentially improve outcomes. On the basis of these studies, many surgeons have modified their intraoperative support techniques. However, review of these studies suggests that the benefit of changing intraoperative support techniques may be less than hoped. Indeed, the findings
TABLE 5. Predictors of head circumference $(\mathbf{n}=188)$

\begin{tabular}{lrcrc}
\hline & Estimate & SE & \multicolumn{1}{c}{$\boldsymbol{t}$} & $\boldsymbol{P r}(>|\boldsymbol{t}|)$ \\
\hline (Intercept) & 29.1072 & 1.8581 & 15.6650 & .0000 \\
Male gender & 1.0262 & 0.2056 & 4.9910 & .0000 \\
Vaginal delivery & -0.6738 & 0.2201 & -3.0610 & .0026 \\
Birth head circumference & 0.3560 & 0.0465 & 7.6550 & .0000 \\
APGAR at 5 min & 0.2300 & 0.1149 & 2.0020 & .0468 \\
Lowest NP temperature & 0.0772 & 0.0353 & 2.1880 & .0287 \\
\hline
\end{tabular}

$S E$, Standard error; $N P$, nasopharyngeal.

of previous studies, as well as the current study, are consistent with the hypothesis that factors (recognized and unrecognized) other than intraoperative management strategies are more important determinants of outcome and explain more of the variability in outcomes.

The neurologic status of children with CHD is often abnormal at birth, before surgical intervention. ${ }^{4,14,15}$ There is increasing evidence that in utero central nervous system development is often abnormal in children with CHD. ${ }^{16}$ Microcephaly and congenital central nervous system malformations are common. Recent studies from our institution have demonstrated that cerebral blood flow is very low in some neonates with uncorrected CHD, often with evidence of cerebral ischemia and preoperative white matter injury characterized by periventricular leukomalacia. ${ }^{17,18}$ Newborn infants with CHD are also exposed to the potential risks of hypoxia, acidosis, and hypotension. Low birth weight is an important predictor of worse outcome. Many factors may contribute to low birth weight, including prematurity, associated genetic syndromes, placental insufficiency, and intrauterine growth restriction, all of which may increase the risk of neurodevelopmental delay.

In addition, genetic factors are a major determinant of neurologic outcome in children with CHD. Many defects are part of well-described syndromes with associated developmental dysfunction, independent of the cardiac defect or cardiac surgery. APOE is an important regulator of cholesterol metabolism. ${ }^{6,7}$ APOE-containing lipoproteins are the primary lipid transport vehicles in the central nervous system. There is increasing evidence that APOE is important for neuronal repair. There are three common isoforms of APOE (E2, E3, and E4), which are encoded by three alleles $(\epsilon 2, \epsilon 3$, and $\epsilon 4$, respectively) and vary by single amino acid substitutions. A strong association has been validated between the APOE $\epsilon 4$ allele and Alzheimer disease. $A P O E$ genotype has been shown to have an important role as a determinant of neurologic recovery after central nervous system ischemia, intracerebral hemorrhage, and traumatic brain injury. There is evidence of an association of $A P O E$ genotype with neurocognitive decline after cardiac surgery in adults and children. ${ }^{6,7}$ The finding that the APOE $\epsilon 2$ allele is associated with a worse outcome is consistent with 
the hypothesis that genetic variants that do not cause CHD may alter the response to environmental factors and thus increase susceptibility to neurologic injury.

Well-designed trials assessing the impact of changes in intraoperative management strategies have often provided conflicting data. In the BCAS, assignment to DHCA was associated with an increased incidence of seizures in the early postoperative period. ${ }^{4}$ Evaluation at 1 year of age demonstrated that the children assigned to DHCA had lower PDI scores. ${ }^{1}$ At 4 years of age, assignment to DHCA was associated with impaired gross and fine motor skills, as well as speech apraxia. ${ }^{2}$ However, cognitive function assessed by full scale, verbal, and performance IQ was significantly lower for the entire cohort compared with the general population, and there was no treatment group effect. Social class predicted a larger percentage of the variation in IQ (24\%) than did treatment group (DHCA vs low-flow CPB) assignment (3\%). Similarly, at the 8-year evaluation, performance of the cohort for many neurodevelopmental domains was worse than population norms, with no effect of treatment group assignment. ${ }^{3}$ However, there were treatment group-specific effects. Motor and speech skills were lower in patients assigned to DHCA, whereas the low-flow bypass group demonstrated worse scores for impulsivity and behavior. As at the 4-year evaluation, social class (23.7\%) and VSD status (3.2\%) explained more of the variance in IQ than did treatment group (DHCA vs low-flow $\mathrm{CPB}$ ) assignment $(0.3 \%)$. Treatment assignment resulted in different neurologic morbidities, rather than reducing their frequency or severity.

As part of a subsequent, prospective, randomized study investigating blood gas management strategies (alpha-stat vs $\mathrm{pH}$-stat) during $\mathrm{CPB}$, investigators at Boston Children's Hospital evaluated neurodevelopmental outcomes at 1 year age. ${ }^{10}$ The investigators concluded that neither strategy was consistently associated with improved or impaired outcomes. PDI scores did not differ between treatment groups. Interestingly, the effect of the blood gas management strategy on MDI scores varied between subgroups on the basis of the cardiac diagnosis. Patients with TGA and TOF tended to have higher MDI scores when randomized to a $\mathrm{pH}$-stat strategy, but the differences were not significant. In the VSD subgroup, patients assigned to an alpha-stat strategy had significantly higher MDI scores than did those assigned to a $\mathrm{pH}$-stat strategy. This variation suggests that the interaction of patient-specific factors (diagnosis) and intraoperative management strategies may modulate outcomes.

A subsequent study evaluated hemodilution during CPB as a potential risk factor for worse neurodevelopmental outcome. ${ }^{11}$ Infants were randomly assigned to a target hematocrit value of $20 \%$ or $30 \%$. At 1 year of age, MDI scores were similar between groups. Significant predictors of MDI score were gender, birth weight, and social class. Assign- ment to the lower hematocrit group was the strongest single predictor of lower PDI scores and accounted for $6.3 \%$ of the variance in PDI scores. However, other patient-specific factors were also independent predictors of PDI scores, including preoperative endotracheal intubation, birth weight, and social class. No other intraoperative management variable, including use and duration of DHCA, was predictive of a worse outcome. Thus, as in the current study, patient-specific factors were important determinants of neurodevelopmental outcomes.

In the current study, as in the BCAS, longer postoperative length of stay was an independent predictor of worse neurodevelopmental outcome. ${ }^{5}$ There is increasing evidence that postoperative events result in central nervous system injury. In a previous study, we evaluated the incidence of periventricular leukomalacia early after neonatal and infant cardiac surgery. Periventricular leukomalacia was identified by early postoperative brain magnetic resonance imaging in more than $50 \%$ of neonates compared with $4 \%$ in patients older than 30 days at the time of surgery. ${ }^{19}$ Hypoxemia and hypotension in the first 48 hours after surgery were associated with an increased occurrence of periventricular leukomalacia. Longer total support time was associated with increased occurrence of periventricular leukomalacia, but not use or duration of DHCA.

\section{Limitations}

This study is a single-center, secondary analysis of a subgroup of an observational study evaluating APOE genotype as a risk factor for worse neurodevelopmental outcomes after infant cardiac surgery. It was not designed as a treatment trial and may be underpowered to determine differences in outcome resulting from changes in intraoperative management strategies, such as hematocrit and DHCA. The patient population is heterogeneous and includes patients with many characteristics, such as genetic anomalies, which may confound attempts to elucidate the impact of intraoperative management strategies. The study group was limited to 2-ventricle CHD repaired in one operation, intending to minimize the effects of chronic cyanosis and multiple operations. Many previous studies have attempted to further minimize confounders by limiting eligibility to a few cardiac defects and excluding low birth weight patients and those with significant extracardiac anomalies. Although this approach may enhance the ability to assess the impact of changes in management, it also significantly limits the generalizability of the findings to the larger population of infants with CHD. The current study is more diverse, in terms of gender, ethnicity, and cardiac diagnosis, than some previous studies. However, because of the exclusion of children with some forms of complex CHD (single ventricle) and those undergoing multiple operations, the results of the current study also may not be generalizable to the larger 
population of patients with CHD. In addition, the intraoperative management strategies were not standardized. Finally, the predictive value of neurodevelopmental testing at 1 year is limited. ${ }^{20}$ The full extent of an early injury often is not completely recognized until long after the event, when complex cognitive and higher executive skills are required. The larger APOE cohort is currently undergoing detailed neurodevelopmental assessment at 4 years of age.

\section{Conclusions}

In this large, heterogeneous cohort of patients undergoing repair of 2-ventricle CHD, patient-specific factors are important determinants of neurodevelopmental outcomes at 1 year of age and contribute more substantially to the risk of adverse neurodevelopmental outcomes than do intraoperative management strategies. Consistent with previous studies, intraoperative management strategies explain only a small portion of the variability in outcomes. Previously described risk factors (both patient-specific and intraoperative variables) explain only part $(<30 \%)$ of the variability in neurodevelopmental outcomes, suggesting that unrecognized factors are important determinants of outcome. Genetic factors are a major determinant of neurologic outcome in children with CHD. Future studies of neurologic outcome and neuroprotective strategies must include risk-stratification for genetic and other patient factors that may alter the risk of central nervous system injury and adverse neurologic outcomes. These factors may be more important determinants of outcome than are intraoperative management strategies. To improve neurodevelopmental outcomes for children with CHD, we must identify and understand the biologic pathways underlying interindividual variation in outcomes and develop individualized, targeted therapeutic strategies.

\section{References}

1. Bellinger DC, Jonas RA, Rappaport LA, Wypij D, Wernovsky G, Kuban KC, et al. Developmental and neurologic status of children after heart surgery with hypothermic circulatory arrest or low-flow cardiopulmonary bypass.[see comment]. N Engl J Med. 1995;332:549-55.

2. Bellinger DC, Wypij D, Kuban KC, Rappaport LA, Hickey PR, Wernovsky G, et al. Developmental and neurological status of children at 4 years of age after heart surgery with hypothermic circulatory arrest or low-flow cardiopulmonary bypass. Circulation. 1999;100:526-32.

3. Bellinger DC, Wypij D, du Plessis AJ, Rappaport LA, Jonas RA, Wernovsky G, et al. Neurodevelopmental status at eight years in children with dextro-transposition of the great arteries: the Boston Circulatory Arrest Trial [see comment]. J Thorac Cardiovasc Surg. 2003;126:1385-96.

4. Newburger JW, Jonas RA, Wernovsky G, Wypij D, Hickey PR, Kuban $\mathrm{KC}$, et al. A comparison of the perioperative neurologic effects of hypothermic circulatory arrest versus low-flow cardiopulmonary bypass in infant heart-surgery. N Engl J Med. 1993;329:1057-64.

5. Newburger JW, Wypij D, Bellinger DC, du Plessis AJ, Kuban KC, Rappaport LA, et al. Length of stay after infant heart surgery is related to cognitive outcome at age 8 years. J Pediatr. 2003;143:67-73.

6. Gaynor JW, Gerdes M, Zackai EH, Bernbaum J, Wernovsky G, Clancy RR, et al. Apolipoprotein E genotype and neurodevelopmental sequelae of infant cardiac surgery. J Thorac Cardiovasc Surg. 2003; 126:1736-45.
7. Tardiff BE, Newman MF, Saunders AM, Strittmatter WJ, Blumenthal JA, White WD, et al. Preliminary report of a genetic basis for cognitive decline after cardiac operations. Ann Thorac Surg. 1997;64:715-20.

8. Hollingshead A. Four factor index of social status. New Haven [CT]. Department of Sociology, Yale University; 1975.

9. Bellinger DC, Rappaport LA, Wypij D, Wernovsky G, Newburger JW. Patterns of developmental dysfunction after surgery during infancy to correct transposition of the great arteries. J Dev Behav Pediatr. 1997; 18:75-83.

10. Bellinger DC, Wypij D, du Plessis AJ, Rappaport LA, Riviello J, Jonas RA, et al. Developmental and neurologic effects of alpha-stat versus $\mathrm{pH}$-stat strategies for deep hypothermic cardiopulmonary bypass in infants [see comment]. J Thorac Cardiovasc Surg. 2001;121:374-83. Erratum in: J Thorac Cardiovasc Surg. 2001;121:893.

11. Jonas RA, Wypij D, Roth SJ, Bellinger DC, Visconti KJ, du Plessis AJ, et al. The influence of hemodilution on outcome after hypothermic cardiopulmonary bypass: results of a randomized trial in infants. J Thorac Cardiovasc Surg. 2003;126:1765-74.

12. Bayley N. Comparisons of mental and motor test-scores for ages 1-15 months by sex, birth-order, race, geographical location, and education of parents. Child Dev. 1965;36:379-411.

13. Lynn R. New data on black infant precocity. Person Indiv Diff. 1998;25:801-4.

14. Limperopoulos C, Majnemer A, Shevell MI, Rosenblatt B, Rohlicek C, Tchervenkov C. Neurodevelopmental status of newborns and infants with congenital heart defects before and after open heart surgery [see comment]. J Pediatr. 2000;137:638-45.

15. Limperopoulos C, Majnemer A, Shevell MI, Rosenblatt B, Rohlicek C, Tchervenkov C. Neurologic status of newborns with congenital heart defects before open heart surgery. Pediatrics. 1999;103:402-8.

16. Glauser TA, Rorke LB, Weinberg PM, Clancy RR. Congenital brain anomalies associated with the hypoplastic left heart syndrome. Pediatrics. 1990;85:984-90.

17. Mahle WT, Tavani F, Zimmerman RA, Nicolson SC, Galli KK, Gaynor JW, et al. An MRI study of neurological injury before and after congenital heart surgery. Circulation. 2002;106(12 Suppl 1):I109-14.

18. Licht DJ, Wang J, Silvestre DW, Nicolson SC, Montenegro LM, Wernovsky G, et al. Preoperative cerebral blood flow is diminished in neonates with severe congenital heart defects. $J$ Thorac Cardiovasc Surg. 2004;128:841-9.

19. Galli KK, Zimmerman RA, Jarvik GP, Wernovsky G, Kuypers MK, Clancy RR, et al., Periventricular leukomalacia is common after neonatal cardiac surgery. J Thorac Cardiovasc Surg. 2004;127:692-704. Erratum in: J Thorac Cardiovasc Surg. 2004;128:498.

20. McGrath E, Wypij D, Rappaport LA, Newburger JW, Bellinger DC. Prediction of IQ and achievement at age 8 years from neurodevelopmental status at age 1 year in children with D-transposition of the great arteries. Pediatrics. 2004;114:e572-6.

\section{Discussion}

Dr Richard A. Jonas (Washington, DC). I congratulate you and your colleagues for providing some data that will help us to unravel the specific impact of patient-related factors on neurodevelopmental outcome before general cardiac surgery.

What you have done is to perform a meta-analysis of a prospective but nonrandomized study of the impact of a specific genotype on neurodevelopmental outcome. Not surprisingly, you found that patient-specific factors were more important than intraoperative manipulations such as the use of circulatory arrest, hematocrit, or $\mathrm{pH}$ strategy.

I say "not surprisingly" because, as you yourself have pointed out in the limitations of the study, this is a very heterogeneous patient population. For example, unlike previous work in this area, you included low birth weight patients and you included patients with known genetic factors and confirmed what is widely known, that both of these factors have an important impact on outcome. 
In addition, you made no attempt in your study to subject patients to a uniform bypass strategy. The duration of cooling before circulatory arrest in some patients was as short as 5 minutes, and the lowest NP temperature recorded before circulatory arrest was as high as $28^{\circ} \mathrm{C}$.

I am concerned that the subliminal message that comes through is that since patient-related factors are so important, we really do not need to focus any attention on remediable intraoperative factors.

Surely you would agree that studies that have attempted to enroll only a very homogeneous patient population and then have subjected patients to a carefully controlled set of intraoperative conditions with variation of only one factor, such as hematocrit or $\mathrm{pH}$, are far more likely to produce specific information regarding the relative importance of intraoperative factors, and the conclusions derived from such studies should be interpreted in that light.

My first question for you is to ask you, once again, why you continue to use the alpha-stat strategy in patients in whom you are considering using circulatory arrest despite the fact that a prospective, randomized trial of $\mathrm{pH}$ strategy documented a significantly higher incidence of perioperative complications, including death to a $P$ value of .06 , when the alpha-stat strategy was used.

I am also interested to know your current strategy regarding hematocrit in patients in whom you intend to use DHCA. What do you consider the current limits of a reasonable standard of care? As we get asked regularly by the lawyers, what is a reasonable standard of care currently for the application of DHCA in terms of duration of cooling, minimum temperature for circulatory arrest, and maximal duration?

Finally, I am interested to know what you believe the next step will be, more specifically, in identifying and understanding the biologic pathways that underlie individual variation in outcomes and how you plan to move to developing individualized targeted therapeutic strategies which, I agree, is certainly going to be a very important development that is coming out of this important work that you are doing.

I certainly do not want to downplay the importance of your focus on patient-specific factors. These clearly are important factors, as we demonstrated in the circulatory arrest study. But I do not think you should use the presence of important patient-specific factors to downplay the fact that there may be differences in outcome according to intraoperative strategies.

Dr Gaynor. First, I think it is important to recognize that even though this is a prospective observational study in which management strategies were not stratified, the findings are very consistent with the other studies such as you mentioned, the hematocrit study, the blood gas study, and the circulatory arrest study. The amount of variability explained by DHCA is very consistent.

In your study of hematocrit, although hematocrit was the single most important factor, you also found that birth weight (even though you excluded low birth weight infants), preoperative intubation, and other factors were significantly important determinants of outcome.

In discussing the alpha-stat versus $\mathrm{pH}$ strategy, there were some perioperative differences in outcome. However, as you acknowledged, neither strategy was associated with improved or impaired neurodevelopmental outcomes.
I pulled out the subgroup of patients who met the criteria for entering the hematocrit study-no genetic syndromes, 2-ventricle heart disease, birth weight greater than $2.3 \mathrm{~kg}$ - and looked at the MDI and PDI for ours with an alpha-stat versus $\mathrm{pH}$ stat. The mean PDI for your group was 85.6 and ours was 85.5 for similar patients. The mean MDI for yours was, I think, 93-point something and ours was 94 . Thus I think the outcomes are very similar, even though we used an alpha-stat and you used a pH-stat strategy, when the patient populations are comparable.

In terms of the cooling for DHCA, there was one patient who had a 1-minute period of circulatory arrest at $28^{\circ} \mathrm{C}$. Everybody else was cooled for at least 15 minutes, and I believe the median was a little bit greater than that. That one patient is an outlier. We do, indeed, cool at least 15 to 20 minutes before the use of circulatory arrest. We have gone to a higher hematocrit on hemodilution. We usually maintain a hematocrit level around 30\% during hemodilution now when we are going to use DHCA.

I think the best data currently out there for duration are the BCAS data, which suggest a safe duration somewhere, with the strategies from the late 1980s, of around 40 minutes. I do not propose that these data will tell us whether there is a safe duration or not.

In the alpha-stat study and in the hematocrit study, approximately $40 \%$ of the patients had circulatory arrest. And circulatory arrest was not a risk factor for outcome in either one of those studies. So I wonder how much effect circulatory arrest has on outcome.

What I think is important is that these studies have provided an incredible amount of information, but I do not know how much variability, how much more improvement in outcome, we are going to get by changing the factors and applying them to the whole population. I think there is good evidence that there are genetic factors that affect the severity-the inflammatory response, coagulation profiles, tolerance to ischemia - which vary significantly across patient populations.

As we begin to understand, we may be able to identify high-risk subgroups, for example, a child who is at a high risk for a hyperinflammatory response or a child who is at risk for microvascular coagulation. I do not know where the factors are going to be. I think we are going to need a genetic association study in much more detail than what I have done with one specific allele. However, if we can identify genetic syndromes and biologic pathways that have relevance to the mechanism of injury, then perhaps if a child produces a lot of tumor necrosis factor- $\alpha$, there may be a monoclonal antibody against tumor necrosis factor- $\alpha$ that will be important for him. If the child is at risk for microvascular coagulation, perhaps we need another type of anticoagulation. I do not know, but I think that is the direction.

What I hope to do with this paper is to say that we need to focus on some of these other factors because I think the amount we are able to change the outcomes with our intraoperative strategies may be limited. It is a testable hypothesis. We have to find out.

Dr Jonas. Regarding the specific changes that you might make, depending on genetic subtype, patients at risk, what exactly would be done?

Dr Gaynor. Well, I think you would have to define. There are some studies in patients with sepsis. If you have identified that they are at risk for a hyperinflammatory response, there are specific 
receptor blockers that can be used to treat them. If we can identify these patients preoperatively, perhaps we should attempt to blunt the inflammatory pathway in that subgroup of patients.

I do not have the specific treatment strategies yet, but I think that is a focus that, as we go forward, we should begin to look at. How are these patients different? One of the things that I found striking in this was the tremendous variability in outcome in the children who had continuous bypass. Even when we eliminated the genetic syndrome patients, we still found the same patient factors outweighed the variability of the operative factors.

Dr John E. Connolly (Irvine, Calif). I congratulate the authors on their interesting findings as likely causes of poor neurodevelopment outcomes. I have several questions.

How did you monitor the infant's temperature and how did you decide how long to cool to obtain what you thought was a safe degree of brain cooling? Did you use ice around the head of the patient?

We have also been interested in the possible causes of supratentorial damage from profound hypothermia and circulatory arrest. To study that, we subjected 80 dogs of various ages to the technique after our psychology department had conditioned them to perform complicated tasks to be repeated after recovery from the hypothermia and arrest. We used direct brain temperatures on those dogs.

We first used deep hypothermia and arrest for open neurosurgical vascular procedures in which we could directly monitor the brain with a thermistor probe in the cortex. We were very impressed with the significant lag between the brain temperature and the body temperature during cooling. Our conclusions from that study were that a brain temperature of $20^{\circ} \mathrm{C}$ is safe for 60 minutes of arrest, but it requires 60 minutes of cooling to reach. For 30 minutes of arrest, we found that we needed 30 minutes of cooling for the brain temperature to reach $28^{\circ} \mathrm{C}$. Our recommendation is that it is always better to cool too long than too short a period of time before arrest.

Dr Gaynor. We used NP temperature as our surrogate for brain temperature. All patients but one were cooled at least 15 minutes to an NP temperature of $18^{\circ} \mathrm{C}$, and we used topical hypothermia to the brain by packing the head in ice.

Dr Connolly. And how long did you cool, just 30 minutes?

Dr Gaynor. At least 15 minutes in all but one patient.

Dr Connolly. Before we had conditioned our dogs, many of the dogs would seem all right and could run around, but this did not demonstrate any damage supratentorially. Therefore, we needed to train the dogs to do some fancy tricks, and then we could assume that there had been absolutely no change in the brain. That is when we concluded that 30 minutes was probably the minimum.
Dr Bradley S. Allen (Houston, Tex). As Dr Fraser and Dr Hanley said yesterday, and Dr Jonas alluded to today, the devil sometimes is in the details. I have two questions.

First, what pressure or flow did you use for your continuous bypass patients? Moreover, did you monitor the pressure, as too high or too low a pressure may be detrimental?

Second, since you saw marked variability in the continuous profusion group, did you look at multiple variables, as Dr Jonas recommended (ie, hematocrit, pressure, flow) to determine whether any of those variables explained the variability in this group?

Dr Gaynor. The pump strategies were not standardized. The pump flow was at the discretion of the surgeon.

We did look at duration of cooling, lowest temperature, and hematocrit. Although I think those are potentially important variables, I do not know of any data that correlate a certain flow or a certain perfusion pressure during continuous bypass to indicate that one pressure or one flow rate is better than another in a human with long-term neurodevelopmental outcome. I do think that is something that could be tested and standardized. It is a testable hypothesis and those studies need to be done.

Dr Tara Karamlou (Toronto, Ontario, Canada). Did you look at socioeconomic status? Did you look at a Hollingshead scale, or how important is that?

Dr Gaynor. We did look at a Hollingshead scale. It did not come out in the analysis, but I think there are some other studies that suggest that at 1 year the environmental influences may not be quite as great. We are now evaluating these children at 4 years, and I will bet the Hollingshead scale will be a significant predictor at 4 years. However, we did include that in the analysis.

Dr Christo I. Tchervenkov (Montreal, Quebec, Canada). It did not come up in the discussion, but what was the preoperative status of the patient? We have shown that $60 \%$ of neonates and $40 \%$ of infants who have undergone a careful examination by a neurologist and neuropsychologist are abnormal preoperatively. Perhaps some of the variability in outcome could be explained by the preoperative neurologic status of these patients. Have you done any preoperative assessments?

Dr Gaynor. I agree entirely. There was not a formal preoperative status, but I think the fact that lower birth weight Apgar scores were associated with the worst outcome suggests that preoperative factors are an important determinant of outcome. Again, back to our hypothesis, it is patient factors, things that exist before and after the operation, as much as or more than what happens in the operating room. 

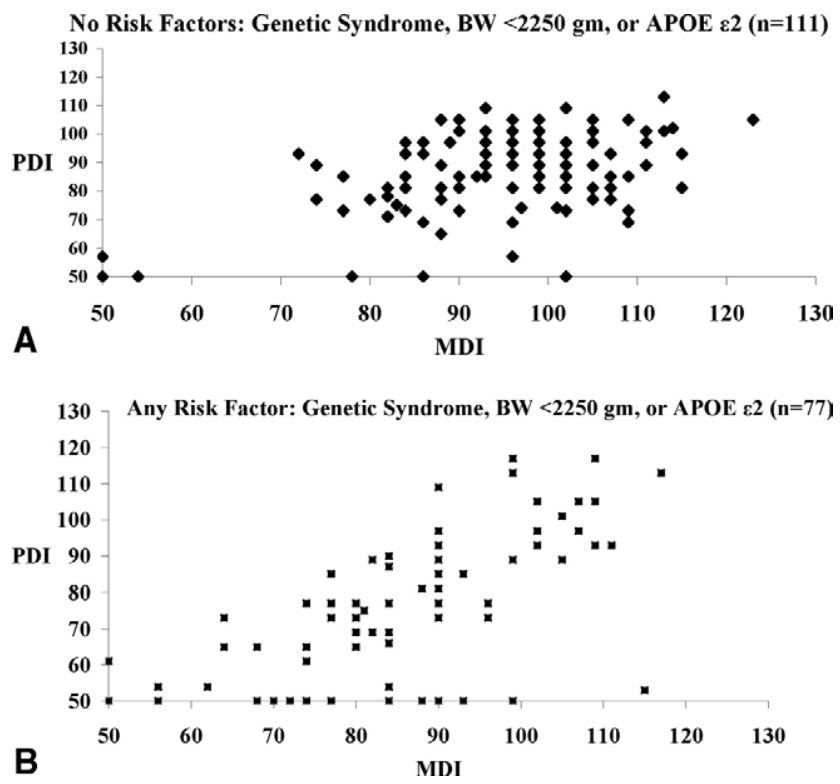

Figure E1. A, X-Y plot showing MDI and PDI scores for 111 patients with no risk factors (low birth weight, genetic syndrome, or APOE $\epsilon 2$ allele). The $x$-axis is the MDI score and the $y$-axis is the PDI score. $B, X-Y$ plot showing MDI and PDI scores for 77 patients with one or more risk factors (low birth weight, genetic syndrome, or APOE E2 allele). The $x$-axis is the MDI score and the $y$-axis is the PDI score. The number of patient with low scores on both measures is increased compared with patients with no risk factors (A). BW, Birth weight; $A P O E$, apolipoprotein E; MDI, Mental Developmental Index; PDI, Psychomotor Developmental Index. 
TABLE E1. Preoperative and intraoperative characteristics of the patients who returned and of those who did not return

\begin{tabular}{|c|c|c|c|c|c|c|c|c|c|c|}
\hline \multirow[b]{2}{*}{ Variable } & \multicolumn{4}{|c|}{ Patients evaluated at 1 year } & \multicolumn{4}{|c|}{ Patients who did not return } & \multirow[b]{2}{*}{ Stat } & \multirow[b]{2}{*}{$\boldsymbol{P}$} \\
\hline & Sample & n & Mean (\%) & SD & Sample & $\mathbf{n}$ & Mean (\%) & SD & & \\
\hline Gender & 188 & & & & 55 & & & & 2.229 & .135 \\
\hline Female & & 85 & $45.2 \%$ & & & 18 & $32.7 \%$ & & & \\
\hline Male & & 103 & $54.8 \%$ & & & 37 & $67.3 \%$ & & & \\
\hline Ethnicity & 188 & & & & 55 & 1 & & & 2.364 & .669 \\
\hline White & & 136 & $72.3 \%$ & & & 35 & $63.6 \%$ & & & \\
\hline Asian/Pacific Islander & & 4 & $2.1 \%$ & & & 1 & $1.8 \%$ & & & \\
\hline Black & & 23 & $12.2 \%$ & & & 11 & $20.0 \%$ & & & \\
\hline Hispanic & & 6 & $3.2 \%$ & & & 2 & $3.6 \%$ & & & \\
\hline American Indian & & 0 & $0.0 \%$ & & & 0 & $0.0 \%$ & & & \\
\hline Other & & 19 & $10.1 \%$ & & & 6 & $10.9 \%$ & & & \\
\hline APOE genotype & 188 & & & & 55 & & & & 2.168 & .826 \\
\hline$A P O E \in 22 / \epsilon 23$ & & 22 & $11.7 \%$ & & & 9 & $16.4 \%$ & & & \\
\hline APOE $\epsilon 33$ & & 117 & $62.2 \%$ & & & 33 & $60.0 \%$ & & & \\
\hline APOE $\epsilon 33 / \epsilon 44$ & & 42 & $22.3 \%$ & & & 10 & $18.2 \%$ & & & \\
\hline APOE $\epsilon 24$ & & 5 & $2.7 \%$ & & & 1 & $1.8 \%$ & & & \\
\hline Unknown & & 2 & $1.1 \%$ & & & 2 & $3.6 \%$ & & & \\
\hline Diagnosis & 188 & & & & 55 & 5 & & & 6.417 & .093 \\
\hline TGA/IVS & & 39 & $20.7 \%$ & & & 5 & $9.1 \%$ & & & \\
\hline VSD & & 34 & $18.1 \%$ & & & 15 & $27.3 \%$ & & & \\
\hline TOF & & 56 & $29.8 \%$ & & & 21 & $38.2 \%$ & & & \\
\hline Other & & 59 & $31.4 \%$ & & & 14 & $25.5 \%$ & & & \\
\hline \multicolumn{11}{|l|}{ Birth history } \\
\hline Type of delivery & 186 & & & & 48 & & & & & \\
\hline Cesarean section & & 129 & $69.4 \%$ & & & 31 & $64.6 \%$ & & 0.211 & .646 \\
\hline Vaginal & & 57 & $30.6 \%$ & & & 17 & $35.4 \%$ & & & \\
\hline Multiple gestation & 187 & & & & 47 & & & & & \\
\hline Yes & & 10 & $5.3 \%$ & & & 0 & $0.0 \%$ & & 74.397 & .000 \\
\hline No & & 177 & $94.7 \%$ & & & 47 & $100 \%$ & & & \\
\hline Gestational age (wk) & 188 & & 38.40 & 2.44 & 49 & & 38.63 & 2.27 & -0.632 & .529 \\
\hline APGAR $1 \mathrm{~min}$ & 184 & & 7.72 & 1.65 & 46 & & 7.93 & 1.36 & -0.906 & .368 \\
\hline APGAR $5 \mathrm{~min}$ & 184 & & 8.63 & 0.94 & 46 & & 8.76 & 0.71 & -1.043 & .300 \\
\hline Birth weight $(\mathrm{g})$ & 187 & & 3107 & 667 & 50 & & 3128 & 630 & -0.213 & .832 \\
\hline Birth length $(\mathrm{cm})$ & 182 & & 49.41 & 3.73 & 46 & & 49.03 & 3.39 & 0.676 & .501 \\
\hline Birth head circumference $(\mathrm{cm})$ & 183 & & 33.47 & 2.18 & 46 & & 33.04 & 2.26 & 1.161 & .250 \\
\hline \multicolumn{11}{|l|}{ Preoperative variables } \\
\hline Intubation & 186 & & & & 48 & & & & & \\
\hline Yes & & 45 & $24.2 \%$ & & & 10 & $20.8 \%$ & & 0.089 & .765 \\
\hline No & & 141 & $75.8 \%$ & & & 38 & $79.2 \%$ & & & \\
\hline Prostaglandin infusion & 186 & & & & 48 & & & & & \\
\hline Yes & & 51 & $27.4 \%$ & & & 9 & $18.8 \%$ & & 1.084 & .298 \\
\hline No & & 135 & $72.4 \%$ & & & 39 & $81.2 \%$ & & & \\
\hline Preoperative LOS (d) & 188 & & 2.15 & 4.92 & 55 & & 1.49 & 2.09 & 1.454 & .147 \\
\hline \multicolumn{11}{|l|}{ Operative variables } \\
\hline Age at operation (d) & 188 & & 61.34 & 54.59 & 55 & & 80.33 & 61.02 & -2.078 & .041 \\
\hline Weight at operation $(\mathrm{kg})$ & 188 & & 4.26 & 1.35 & 55 & & 4.39 & 1.30 & -0.641 & .523 \\
\hline Total support time (min) & 188 & & 86.99 & 43.47 & 55 & & 73.85 & 28.07 & 2.660 & .009 \\
\hline CPB duration (min) & 188 & & 75.32 & 46.63 & 55 & & 63.58 & 29.61 & 2.239 & .027 \\
\hline Use of DHCA & 188 & & & & 55 & & & & 0.546 & .460 \\
\hline Yes & & 67 & $35.6 \%$ & & & & 16 & $29.1 \%$ & & \\
\hline No & & 121 & $64.4 \%$ & & & & 39 & $70.1 \%$ & & \\
\hline DHCA duration (min) & 67 & & 32.7 & 14.8 & 16 & & 35.3 & 10.3 & -0.819 & .419 \\
\hline Cooling time (min) & 188 & & 12.40 & 6.09 & 55 & & 12.58 & 7.07 & -0.174 & .862 \\
\hline Lowest NP temperature $\left({ }^{\circ} \mathrm{C}\right)$ & 188 & & 22.80 & 5.30 & 55 & & 23.93 & 5.02 & -1.442 & .153 \\
\hline Hematocrit after hemodilution (\%) & 188 & & 27.22 & 4.15 & 55 & & 27.53 & 3.98 & -0.502 & .617 \\
\hline
\end{tabular}


TABLE E1. Continued

\begin{tabular}{|c|c|c|c|c|c|c|c|c|c|c|}
\hline \multirow[b]{2}{*}{ Variable } & \multicolumn{4}{|c|}{ Patients evaluated at 1 year } & \multicolumn{4}{|c|}{ Patients who did not return } & \multirow[b]{2}{*}{ Stat } & \multirow[b]{2}{*}{$\boldsymbol{P}$} \\
\hline & Sample & $\mathbf{n}$ & Mean (\%) & SD & Sample & $\mathbf{n}$ & Mean $(\%)$ & SD & & \\
\hline ECMO/VAD & 188 & & & & 55 & & & & & \\
\hline Yes & & 2 & $1.1 \%$ & & & 0 & $0.0 \%$ & & 104.292 & .000 \\
\hline No & & 186 & $98.9 \%$ & & & 55 & $100 \%$ & & & \\
\hline Delayed sternal closure & 188 & & & & 55 & & & & 70.719 & .000 \\
\hline Yes & & 13 & $6.9 \%$ & & & 0 & $0.0 \%$ & & & \\
\hline No & & 175 & $93.1 \%$ & & & 55 & $100 \%$ & & & \\
\hline \multicolumn{11}{|l|}{ Postoperative variables } \\
\hline Total hospital LOS (d) & 188 & & 10.91 & 13.02 & 55 & & 8.40 & 6.77 & 1.909 & .058 \\
\hline Postoperative LOS (d) & 188 & & 8.76 & 10.73 & 55 & & 6.91 & 5.56 & 1.708 & .089 \\
\hline
\end{tabular}

